\title{
Saving the third generation from HIV; first case report in Sri Lanka
}

\author{
D. A. Karawita, J. K. Premadasa, L. P. Dharmasiri, T. Guruparan
}

\begin{abstract}
A 20-year-old unmarried HIV positive female patient presented to the HIV clinic, Colombo with oral candidiasis, generalized pruritic papular eruptions (PPE), weight loss and intermittent fever for 6 months' duration. Her CD4 count was 50 cells/ $\mu$ l. This patient was first found to be HIV antibody positive at the age of 8 years, when she was screened due to her mother's HIV positive status.

Following the presentation, patient was started on ART in December 2010 and continued for 3 years. After that the patient defaulted for about 3 months and re-attended the clinic. At that visit she was pregnant with 20 weeks of gestation. Her partner was HIV negative. Later the patient gave birth to an HIV negative boy with the support of the national programme on Prevention of Mother-to-Child Transmission of HIV (PMTCT) in Sri Lanka. Mother of the index patient and the index patient represent the $1^{\text {st }}$ and $2^{\text {nd }}$ generation while the third generation infection was prevented with PMTCT intervention.
\end{abstract}

Key words: First case, Third generation, mother to child transmission, HIV, Sri Lanka

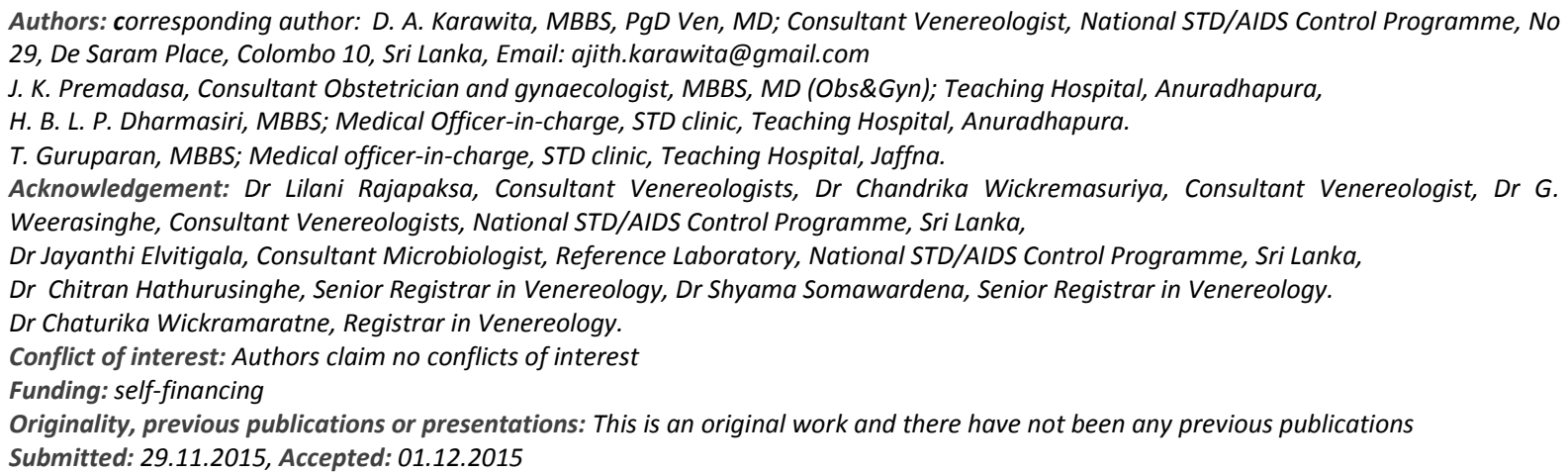

\section{Full article}

Introduction

Infants can acquire Human Immunodeficiency Virus (HIV) infection from their infected mother during pregnancy, delivery (child birth) and breastfeeding. Mother to child transmission (MTCT) of HIV is the commonest way of children getting infected with HIV. Globally, an estimate of 2.6 million children (<15 years) are living with HIV and 220,000

children are estimated to be newly infected with HIV in 2014 (1). In Sri Lanka, as of end 2014, it is estimated that 100 children $(<15$ years) are living with HIV although 71 mother to child infections reported to the National STD/AIDS Control Programme (NSACP) (2).

Sri Lanka has started the prevention of Mother-to-Child Transmission of HIV programme in 2002. The elimination of MTCT of HIV programme was launched in 2013. Successful implementation of national programme on the Prevention of Mother to Child Transmission (PMTCT) of HIV can lead to elimination status. WHO has already identified Sri Lanka as one of the potential countries in the region to eliminate MTCT of HIV. Therefore, Sri Lanka is in the process of implementing the EMTCT programme to 
achieve and qualify WHO validation targets of EMTCT. The minimum EMTCT impact targets for HIV identified for Sri Lanka are $<50$ new paediatric infections per 100,000 live births (3).

In this background, we describe a success story of prevention of mother to child transmission of HIV in the third generation of a family. The management of this patient was carried out at different centres, such as Teaching Hospital, Jaffna, National Hospital of Sri Lanka (NHSL), NSACP Colombo and STD clinic, Anuradhapura.

\section{Case history}

The patient was 20 years at the time of presentation in November 2010 (Date of Birth 20.09.1990) and initially presented to the Teaching Hospital, Jaffna with oral candidiasis, generalized Papular Pruritic Eruptions (PPE) with weight loss and intermittent fever for 6 months duration. Then the patient transferred to the NHSL and to the HIV clinic, Colombo for further management. At the HIV disease evaluation, her CD4+ lymphocyte count was 50 cells $/ \mu \mathrm{l}, \mathrm{BMI}-17.2 \mathrm{Kgcm}^{2}$ (Height $-148.5 \mathrm{~cm}$, Weight-38kg), WHO clinical stage - IV and performance scale was "A" (Normal Activity).

She had been diagnosed as having HIV in 1998 (at 8 years of age), during a family screening in India when her mother was tested for HIV due to an unusual trophic ulcer in the lower limb. Index patient's father also found to be positive for HIV and all three family members had been started on ART in 1999 in India. But they have defaulted treatment after 9 months of commencing ART treatment.

In 2004, family returned to Sri Lanka but did not attend for HIV services. In 2005, mother of the index patient died of septicaemia at Teaching Hospital, Jaffna.

At HIV clinic, Colombo, antiretroviral therapy (ART) was started for the index patient in December 2010 with tenofovir, emtricitabine and efavirenz (TDF+FTC+EFV). ART was subsequently changed to zidovudine, emtricitabine and efavirenz (ZDV+FTC+EFV) due to an ART drug stock out issue. After 9 months of treatment at the HIV ClinicColombo, her Viral load became undetectable and the regimen was further continued for 3 years (till December 2013), after which she defaulted HIV treatment.

The patient re-attended the Colombo HIV clinic in March 2014. She was pregnant with 20 weeks of gestation. Her last menstrual period was (LMP) on 22.10.2013 and the expected date of delivery (EDD) was on 29.07.2014. The same previous regimen of ZDV+3TC+EFV was started on 24.03.2014 and investigations done at 22 weeks of gestation, indicated that her Viral Load was 873 copies/ml (COBAS TaqMan HIV-1 Test V 2.0), CD4+ lymphocyte count was 434 cells/ $\mu$ l and haemoglobin was $10.8 \mathrm{mg} / \mathrm{dl}$.

Patient wanted to attend the STD clinic, Teaching Hospital (TH), Anuradhapura as it is close to her home town for the delivery. Therefore, patent was transferred to the $\mathrm{TH}$, Anuradhapura at POA of 32 weeks. There, the ART regimen was changed to tenofovir, emtricitabine, and boosted lopinavir $(\mathrm{TDF}+\mathrm{FTC}+\mathrm{LPV} / \mathrm{r})$ at 32 weeks of gestation. The patient was referred to a consultant obstetrician \& gynaecologist for the management of the delivery. Dates were confirmed by USS and an elective Lower Segment Caesarean Section (LSCS) was scheduled on 11.07.2014 (POA = 38 wks). ART was continued and a day before the elective LSCS the viral load was 307 copies/mL (Log value 2.48), CD4+ lymphocyte count was 543 cells/ $\mu \mathrm{l}, \mathrm{CD} 8-2000$ cells/ $\mu \mathrm{l}$ and CD4/CD8 ratio0.27. On 11.07.2014 baby boy was delivered by elective LSCS at the Teaching Hospital, Anuradhapura. 


\section{Management of the neonate}

Precautions were taken to reduce the chance of possible infections during the procedure by creating a less blood field such as early clamping of the cord, sucking of secretions and cleaning of eyes and mucous membranes. Neonate was $2640 \mathrm{~g}$ at delivery. Neonate was asymptomatic and based on the weight band, infant prophylaxis with $10 \mathrm{mg}$ once-daily nevirapine (NVP) was commenced and continued for 6 weeks. Breast feeding was avoided and formula feeding was arranged with EMTCT programme support. Once the prophylaxis regimen was over, sample of infant blood sent for Early Infant Diagnosis (EID) to India for DNA Nested PCR at 3 months of age and another sample at the age of 12 months and both became negative. Ideal timing of testing could not be followed due to logistical issues.

Bacillus Calmette-Guerin (BCG) vaccine was avoided and the live oral polio vaccine (OPV) was replaced with polio vaccine. Deferred BCG vaccination given after the second negative HIV DNA PCR performed at 12 months of age.

\section{Partner management}

This is a discordant couple of HIV infection and the couple was counselled during the pregnancy for safe sex, consistent use of condoms and post exposure prophylaxis. HIV ELISA (Combo) tests were done on husband before and after the delivery and all tests gave negative results.

\section{Mode of acquisition of HIV by the patient}

According to the history given by the father of the index patient, he had multiple heterosexual contacts when he had been sailing in France, Italy, Russia, Germany, Canada, UK, Holland and Spain during the period of 1977-1982 which is the early phase of the HIV epidemic in the world. Father's acquisition of HIV was due to heterosexual contacts with multiple partners.

In 1982, he had returned to Sri Lanka and got married. Then the family got two daughters; elder one was born in 1984 and the younger (Index patient) was born in India in 1990 when the family was in India during the conflict period in Sri Lanka. Index patient's mother had acquired the infection from her father.

The most probable mode of acquisition of HIV in the index patient would have been from her infected mother and she was found to be infected at the age of 8 years while her family was screened for HIV. Family tree and the infected members are outlined in the figure 1.

\section{Figure 1: Family tree and infected members}

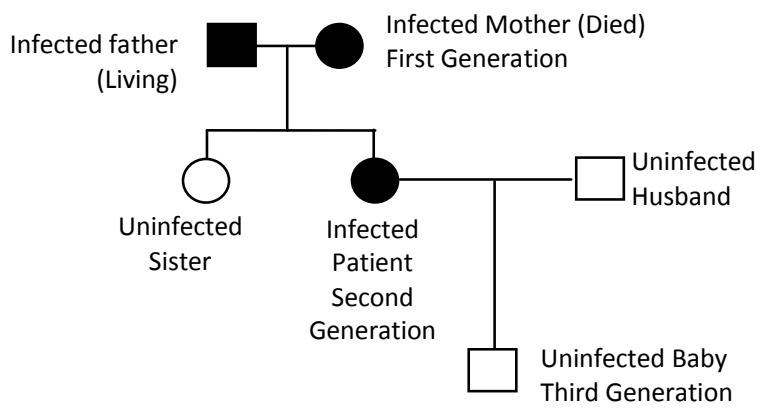

\section{Discussion}

There is a probability that a newborn can acquire HIV infection from their infected mother during pregnancy, delivery and breastfeeding. In the absence of any interventions, transmission risk is $15-30 \%$ in non-breast feeding populations (4). Breast feeding by an infected mother adds an additional $5-20 \%$ risk for an overall transmission rate of $20-45 \%$ (4).

There are number of associated factors that can increase the probability of MTCT of HIV. Strength of association is strong with maternal viral load, immune deficiency, AIDS, duration of membrane rupture, mode of delivery, invasive procedures, prematurity, breast feeding and breast health. Strength of 
association is medium with regard to sexually transmitted infections (STIs) and chorioamnionitis (5).

Avoidance or reduction of exposure to risk factors during the natural course of pregnancy, delivery and breastfeeding was the main target of prevention of MTCT in an already infected mother. Ideally it is expected to achieve undetectable level of viral load at delivery. According to the PMTCT guidelines of WHO following four interventions were carried out.

\section{Maternal antiretroviral therapy \\ 2. Planned caesarean section \\ 3. Infant prophylaxis \\ 4. Formula feeding}

In addition, exposures to other potential risk factors were minimized especially procedural exposures at the caesarean section such as exposure of the foetal mucous membranes to maternal blood or bloody fluids. Effect of maternal antiretroviral therapy on the suppression of maternal viremia was not optimum at the time of the LSCS (Viral Load was 307 copies/ml with CD4+ lymphocyte count was 543 cells/ $\mu \mathrm{l}$ ). According to the available data adequate viral suppression (undetectable) had been achieved 9 months after starting ART in December 2010 about 3 years before the LMP. The next Viral Load test was available on 24.03.2014 (at 22 weeks of pregnancy) and it was 873 copies $/ \mathrm{ml}$ and CD4+ lymphocyte count was 434 cells/ $\mu$ l. From 22 weeks to delivery (at 37 weeks and 3 days) VL was probably between 873 to 307 copies $/ \mathrm{mL}$ while CD4+lymphocyte count varied from $434-543$ cells $/ \mu$ l. If there had been no prevention interventions, chance of transmission would have been approximately $17 \%$ (6). This chance could have been reduced by the use of ART during the patient's pregnancy although the viral suppression was not optimal.

Pre-planned LSCS lowered the rate of HIV-1 perinatal transmission (7) but data are insufficient to evaluate the potential benefit when HIV-RNA levels below 1000 copies/ml.
However, incidence of postnatal morbidity is high with LSCS than vaginal delivery.

Infant prophylaxis with once-daily nevirapine (once daily-NVP) can further reduce the chance of transmission. Studies done among breast feeding populations showed that once daily-NVP can reduce the transmission by $54 \%$ (8).

Formula feeding with complete avoidance of breast feeding can reduce about $5-20 \%$ chance of transmission (4). With the overall interventions and the natural chance of noninfection prevented the baby being infected and saved the third generation of the infected family.

\section{Reference}

1 UNAIDS. United National Joint Programme on AIDS (UNAIDS). [Online].; 2015 [cited 2015 November 08. Available from: http://www.unaids.org/en/resources/

2 National STD/AIDS Control Programme. [Online].; 2014 [cited 2015 November 08. Available from: http://www.aidscontrol.gov.lk/web/images/pdf/quart erly reports.

3 WHO. Global Guidance on Criteria and Processes for Validation: Elimination of Mother-to-Child Transmission of HIV and Syphilis Geneva: WHO; 2014.

4 De Cock, K.M. et al. Prevention of mother-to-child HIV transmission in resource-poor countries:translating research into policy and practice. JAMA. 2000; 283(9): p. 1175-82.

5 Marie-Louise Newell. Prevention of mother-to-child transmissionof HIV: challenges for the current decade. Bulletin of the World Health Organization. 2001: p. 1138-44.

6 Athena P Kourtis, Francis K Lee, Elaine J Abrams, Denise J Jamieson, Marc Bulterys. Mother-to-child transmission of HIV-1: timing and implications for prevention. Lancet Infect Dis. 2006;(6): p. 726-32.

7 Read JS, Newell ML. Efficacy and safety of cesarean delivery for prevention of mother-to-child transmission of HIV-1. The Cochrane Database of Systematic Reviews. 2005;(4).

8 Coovadia HM1, Brown ER, Fowler MG, Chipato T, Moodley D, Manji $\mathrm{K}$ et al. Efficacy and safety of an extended nevirapine regimen in infant children of breastfeeding mothers with HIV-1 infection for prevention of postnatal HIV-1 transmission (HPTN 046): a randomised, double-blind, placebo-controlled trial. Lancet. 2012 Jan; 379(9812): p. 221-8.

9 Epidemiology Unit. Epidemiology Unit, Ministry of Health. [Online].; June 2011 [cited 2015 November 08. Available from: http://www.epid.gov.lk. 